\section{Severe Acute Respiratory Syndrome Coronavirus-2 Induces Cytokine Storm and Inflammation During Coronavirus Disease 19: Perspectives and Possible Therapeutic Approaches}

\author{
Federica Mannino, Alessandra Bitto * and Natasha Irrera \\ Department of Clinical and Experimental Medicine, University of Messina, Messina, Italy
}

The new coronavirus outbreak was first identified in Wuhan, China, in December 2019, and has turned out to be a global health emergency, affecting millions of people worldwide. Coronavirus disease 19 (COVID-19), caused by the SARS-CoV-2 virus, can manifest with flu-like symptoms and can be complicated by severe pneumonia with acute respiratory distress syndrome (ARDS); however a large percentage of infected individuals do not have symptoms but contribute to the spread of the disease. Severe acute respiratory syndrome coronavirus-2 infection has become a global public health emergency since no available treatment seems effective and it is hard to manage the several complications caused by an intense release of cytokines. This paper reviews the current options on drugs used to reduce the deadly effects of the cytokine storm.

Keywords: severe acute respiratory syndrome coronavirus, cytokine storm, inflammation, coronavirus disease, Therapeutics

\section{INTRODUCTION}

Every year diseases of viral origin that affect the respiratory tract represent an important problem of public health. The severe acute respiratory syndrome coronavirus (SARS-CoV) in 2002-2003, H1N1 influenza in 2009, and the Middle East respiratory syndrome coronavirus (MERS-CoV) in 2012 significantly increased the rate of mortality among the population worldwide. In March 2020, the WHO declared the coronavirus disease 19 (COVID-19) as a pandemic worldwide that represents a serious public health risk. SARS-CoV, MERS-CoV, and SARS-CoV-2 belong to the coronaviruses class. Coronaviruses are enveloped viruses characterized by a coronary appearance and positive single-stranded RNA genome (Fung and Liu, 2019) that, crossing species barriers, cause illness starting from the "common" cold to more severe diseases.

Patients may show different signs and symptoms that make the differential diagnosis hard for general practitioners; thus the swab sampling remains the crucial point for diagnosis. The most common symptoms include rhinorrhea, fever, nonproductive cough, loss of taste or smell, myalgia, dyspnea, fatigue, and alteration of the leukocyte count (Chakraborty et al., 2020).

Coronaviruses probably originated from bats and then moved to other hosts. In particular, SARS$\mathrm{CoV}$ and MERS-CoV, respectively, infected the Himalayan palm civet and the dromedary camel, before reaching humans, whereas the exact dynamic transmission of SARS-CoV-2 is still unknown, even if the probability of an animal origin is very high, including snakes, birds, marmots, and bats (Chakraborty et al., 2020). 
Coronavirus-19 (SARS-CoV-2) is particularly infectious to humans, seriously affects the respiratory tract, and causes the COVID-19 (Du Toit, 2020). The high susceptibility of the population is responsible for the raised incidence of the distribution worldwide so that also COVID-19 has become a serious public health problem.

Coronaviruses, and also SARS-CoV-2, are named "corona" because they have a crown-like appearance if observed under electron microscopy; therefore the term "coronam," the Latin equivalent of "crown," has been used. In particular, the spike glycoproteins on the envelope of the virus confer the typical shape of a crown (Chan et al., 2013), and both the SARS-CoV-2 pathophysiology and virulence are related to structural and nonstructural proteins (NSPS). NSPS may act by blocking the host innate immune response whereas the structural proteins contribute to viral assembly and release (Lei et al., 2018). The spike glycoproteins of the crown structure are organized in two subunits, S1 and S2, that may link each other creating homotrimers that, in turn, bind the host receptors (Song et al., 2018). The $S 2$ is the highly conserved subunit and, for this reason, represents the target of the antiviral drugs (anti-S2), whereas the spike receptor-binding domain shows a similarity of about $40 \%$ to that of the SARS-CoVs. The other structures, such as the open reading frame (ORF) $3 \mathrm{~b}$, and the protein encoded by ORF 8 have no homology with the other SARS-CoVs (Cascella et al., 2020).

SARS-CoV-2, as SARS-CoV, specifically binds angiotensinconverting enzyme 2 (ACE2) (Li et al., 2005), using this as the port of entry into the cells. However, SARS-CoV-2 contains some structural differences in the hACE2-binding ridge compared to SARS-CoV, such as the residues 482-485: Gly-Val-Glu-Gly, that increase the binding affinity (Shang et al., 2020).

Therefore, lung damage and inflammation primarily start following the specifying binding to ACE2, but probably it is not the only viral mechanism (Behl et al., 2020; Bordallo et al., 2020; Verdecchia et al., 2020). The exuberant inflammation may certainly be considered as one of the main causes of severity and mortality during SARS-CoV-2 infection.

The aim of this paper is to focus on the possible inflammatory mechanisms mediated by SARS-CoV-2 and the possible therapeutic approaches that might ameliorate the prognosis of the disease, thus reducing the rate of mortality.

\section{SEVERE ACUTE RESPIRATORY SYNDROME CORONAVIRUS-2 AND CYTOKINE STORM}

SARS-CoV-2 infects the respiratory tract, causing an acute lung injury also characterized by an aggressive inflammatory response (Wong et al., 2004). COVID-19 severity is often related to the exacerbation of the inflammatory process; in fact, high concentrations of cytokines [interleukin (IL)-2, IL-7, IL-10, GCSF, IP10, MCP1, MIP1A, TNFa] were observed in plasma of 13 patients admitted to the intensive care unit (ICU) of Wuhan in China. However, also non-ICU patients (n 28) showed increased plasma levels of IL-1 $\beta$, IFN-c, IP-10, MCP-1, IL-4, and IL-10 even if the "cytokine storm" was higher in patients with a severe COVID-19 (Huang et al., 2020).

The increased levels of cytokines and chemokines amplify the innate immune response and stimulate the monocytemacrophages, neutrophils, NK cells, and eosinophils recruitment, thus worsening tissue damage related to the acute respiratory distress syndrome: proinflammatory cytokines levels and severity of the disease are strictly linked (Beacon et al., 2020).

Both the excessive immune reaction and the "cytokine storm" caused by the viral infection result in toxicity and respiratory depression up to pulmonary tissue damage which is the leading cause of mortality in COVID-19. One of the main actors of the cytokine storm is IL-6, which, once released by activated leukocytes, stimulates the production of the acute phase proteins, regulates the thermogenesis, and concurs to the cytokine release syndrome characterized by fever and multiple organ dysfunction (Cascella et al., 2020).

Two inflammatory phases may be described during COVID19: a primary inflammatory response and a secondary inflammatory response. Primary inflammatory response precedes the production of neutralizing antibodies (NAb) and is mainly related to viral replication, viral-mediated ACE2 downregulation, and also the first host response. Secondary inflammatory response occurs when the adaptive immunity is activated and NAb are produced (Fu et al., 2020).

The activation of apoptosis in the epithelial and endothelial cells and pyroptosis in macrophages and lymphocytes leads to the release of proinflammatory cytokines (Yang, 2020).

Previous studies have demonstrated that acute lung injury may be associated with the loss of function of ACE2, which increases vascular permeability and pulmonary edema (Imai et al., 2005). As described, SARS-CoV-2, as SARS-CoV, mediates its entry inside cells through ACE-2 in association with S-protein priming by the host cell protease TMPRSS2; SARS-CoV-2 can downregulate ACE2 and, in turn, could decrease the angiotensin-II clearance, thus worsening tissue damage (Wang et al., 2008; Glowacka et al., 2010; Chatterjee et al., 2020). ACE2 function, in fact, is strictly related to the renin-angiotensin system (RAS): downregulation of ACE2 causes RAS dysfunction, thus increasing inflammation.

The secondary inflammatory response is related to the production of antibodies to face viral infection. The presence of anti-S-neutralizing antibodies in patients highly correlates with the outcome of the disease (Zhang et al., 2006). Fc receptors (FcR) blockade reduces proinflammatory cytokines release but when the virus-anti-S-IgG (anti-spike antibodies) complex binds to the FcR, an inflammatory response is promoted; therefore, anti-S-IgG may be considered as additional activators of inflammation (Liu et al., 2019).

\section{NLR FAMILY PYRIN DOMAIN CONTAINING 3 INFLAMMASOME IS ACTIVATED FOLLOWING THE TOLL-LIKE RECEPTOR STIMULATION}

SARS-CoV-2 may also bind the Toll-like receptors (TLRs) which are membrane receptors also found in immune cells, in $\mathrm{T}$ and 
B cells and in macrophages. Both the TLR 5 and TLR7, for example, are recognized by the sensing single-stranded RNA viruses (Kumar et al., 2009).

Once stimulated, TLR interacts with the intracellular Toll/IL-1 receptor (TIR) domain, inducing a pathway that may activate AP$1, N F-k B$, and IRF3/7 that, in turn, promote the production of cytokines, chemokines, and type I interferons (IFNs) (Takeuchi \& Akira, 2010).

In fact, since TLR5 modulation might play a protective role against respiratory infection, it has been hypothesized that the TLR5 immunomodulation might reduce cytokine storm production and restore the altered immune response following SARS-CoV-2 infection, even if this hypothesis has to be fully investigated (Chakraborty et al., 2020).

It has been recognized that SARS-CoV binds the TLR7, thus inducing the production of proinflammatory cytokines, such as TNF-a, IL-6, and IL-12 (Li et al., 2013), even if SARS-CoV effects following TLR7 activation still need to be investigated. A previous study showed that the use of a SARS coronavirus papain-like protease may inhibit the TLR7 pathway, thus reducing the activation of the transcription factors IRF3, NF- $\mathrm{BB}$, and c-Jun and consequently IFN- $\alpha$, IFN- $\beta$, TNF- $\alpha$, IL- 6 , and IL- 8 production (Li et al., 2016). SARS-CoV and SARS-CoV-2 show similarity; in fact, SARS-CoV-2 genome also contains fragments that could be recognized by TLR7/8, even if the affinity of SARS-CoV-2 on the TLR7 has not been confirmed yet (Moreno-Eutimio et al., 2020). TLR7 stimulation may induce IFN-regulated cytokines release and, interestingly, SARS-CoV-2, binding the TLR, may promote NLRP3 inflammasome activation (Conti et al., 2020), further inducing an inflammatory response and worsening the prognosis of COVID-19. The mechanism that describes how the TLR receptor may activate the NLRP3 inflammasome is related to the pattern recognition receptors (PRRs) (Kawai \& Akira, 2010). Inflammasomes are activated by pathogen associated molecular patterns and damage associated molecular patterns, and once activated, on one hand they stimulate the production and the release of proinflammatory cytokines, and on the other hand they activate additional innate immune cells (Iwasaki and Medzhitov, 2015). TLRs activation following the interaction with a microbial agent causes the production of a supramolecular organizing center (SMOC), called myddosome, in cytoplasm. Myddosome stimulates the activation of the transcription factors involved in the production of proinflammatory cytokines: NF- $\mathrm{kB}$ and AP-1 (Kagan et al., 2014).

However, among the proinflammatory cytokines, the IL-1 pathway follows a different paradigm: PRRs activates IL-1 signaling, thus promoting the production of a SMOC called inflammasome (Martinon et al., 2002).

In particular, NLRP3 inflammasome is composed by NLRP3, apoptotic speck-containing protein adaptor, and procaspase-1. Procaspase-1 is cleaved into caspase-1 following NLRP3 activation and caspase- 1 mediates the release of IL- $1 \beta$ and IL18 (Martinon, 2010). SARS-Cov2 induces the production of IL- $1 \beta$ which is a mediator of lung inflammation, fever, and fibrosis, following TLR stimulation and inflammasome activation, thus contributing with an "additional via" to the cytokine storm during COVID-19.

\section{THERAPEUTIC APPROACHES FOR THE TREATMENT OF SEVERE ACUTE RESPIRATORY SYNDROME CORONAVIRUS-2 CYTOKINE STORM}

Nowadays neither a specific treatment nor a vaccine has been found to manage SARS-CoV-2 infection; therefore the attention of the researchers worldwide is focused on the identification of an effective molecule to treat COVID-19.

Recent reports showed that more than $85 \%$ of patients with peculiar symptoms (fever, cough, and shortness of breath) received antiviral drugs, such as lopinavir and ritonavir tablets (400/100 mg twice a day per os) or remdesivir (once a day by intravenous injection) to ameliorate severe disease outcomes (Chen N. et al., 2020; Holshue et al., 2020; Li and De Clercq, 2020). These drugs have been introduced into COVID-19 treatment based on a virtual screening that identified lopinavir, an aspartate protease inhibitor, as a possible inhibitor of SARS-CoV-2 protease; ritonavir is usually administered with it since its inhibitory action on cytochrome P450 increases lopinavir plasma half-life. Unfortunately, more recently (Cao et al., 2020) a trial on 199 patients comparing the antiviral combination with standard treatment demonstrated no difference in the time to clinical improvement or mortality. On the other hand, remdesivir (GS-5734) has been approved by FDA for treating hospitalized COVID-19 patients on May 1, 2020. Remdesivir is an adenosine analog developed for Ebola and Marburg virus infection and its mechanism of action is based on the interaction with the viral RNA-dependent RNA polymerase thus reducing viral RNA production: in particular, the triphosphate form of remdesivir inhibits the viral RNA synthesis exploiting a specific mechanism of delayed chain termination (Saha et al., 2020a). Few weeks later a multicenter trial carried out in Hubei, China, on 158 patients treated with remdesivir versus 79 with standard care, failed to demonstrate a reduced time to clinical improvement or mortality, with a higher incidence of serious adverse events (Wang et al., 2020). In this scenario, another drug with strong anti-inflammatory effect and low interaction with plasma proteins and the CYP-P450 enzymes, baricitinib, has been proposed to be used in association with the antivirals lopinavir, ritonavir, and remdesivir (Stebbing et al., 2020). The rationale for baricitinib use relies on its mechanism of action; the drug is a Janus kinase (JAK) inhibitor, which blocks JAK-STAT pathway and the consequent release of cytokines. Usually, viral infection causes the release of type I IFN which interacting with its specific receptor induces JAK-STAT pathway activation causing a significant increase of circulating cytokines (Yu et al., 2017). Since the inflammatory response represents one of the major causes of lung damage and mortality, it was speculated that JAK inhibition may be useful to potentiate antivirals effect adding anti-inflammatory effects (Richardson et al., 2020). There are currently four clinical trials in Italy (NCT04320277), Spain (NCT04346147), and US (NCT04340232, NCT 04401579), evaluating the effect of baricitinib alone or in combination with antiviral drugs and the results are expected soon. 
Other nucleoside analogs, such as favipiravir and ribavirin, are also used to treat viral infections of respiratory tract (Jordan et al., 2018) and might be effective for SARS-CoV-2. In fact, nucleoside analogs which derivate from adenine or guanine act on the RNAdependent RNA polymerase, thus inhibiting viral RNA synthesis. Their efficacy remains uncertain because of their nonspecific action against SARS-CoV-2 and because of the possible appearance of side effects, such as anemia at high doses ( $\mathrm{Li}$ and De Clercq, 2020). However, in a recent trial ribavirin was used in combination with lopinavir/ritonavir and interferon beta$1 \mathrm{~b}$ with good results in reducing the time to negative swab (Hung et al., 2020), while the combinations of i) favipiravir and IFN- $\alpha$ and ii) favipiravir and baloxavir marboxil are currently under investigation in patients with COVID-19. It is important to note that the use of antivirals represents a nonspecific approach to treat SARS-CoV-2 since coronaviruses have different proteases: a possible new therapeutic strategy might be to synthesize a specific (i.e., a cysteine protease inhibitor) or a wide spectrum inhibitor.

At the present moment, also other therapeutic approaches are controversial; for many weeks chloroquine and hydroxychloroquine, widely used for the treatment and the prevention of malaria, have been also administered in patients affected by COVID-19. As for SARS-CoV and H5N1, chloroquine inhibits SARS-CoV-2 entry into the cells by glycosylating ACE2 receptors (Vincent et al., 2005); clinical trials showed that it may reduce the number of hospitalizations, ameliorating patient outcomes (Savarino et al., 2006; Yan et al., 2013). Also WHO included these antimalarial as treatments in the SOLIDARITY trial (NCT04330690), but very recently a multinational registry analysis on 96,032 patients around the globe failed to demonstrate a real benefit of hydroxychloroquine or chloroquine used alone or with a macrolide on the final outcome (Mehra et al., 2020). Among the other antiviral strategies, umifenovir (Arbidol) is a new antiviral option approved in China for the management of COVID-19: it prevents virus entry by binding to hemagglutinin (Aktas et al., 2020). However, clinical studies on umifenovir showed that the administration of umifenovir alone was ineffective, whereas the combination with other antivirals might ameliorate viral clearance and improve lung damage (Chen et al., 2020; Song et al., 2020).

Besides ACE2 binding, SARS-CoV-2 exploits the serine protease TMPRSS2 for S-protein priming; therefore, the use of a TMPRSS2 inhibitor, such as camostat mesylate, might inhibit SARS-CoV-2 infection (Hoffmann et al., 2020); however there are no results available from the clinical trials that have been registered in Israel (NCT04355052), Denmark (NCT04321096), and Kentucky (NCT04374019) although they might have promising results since TMPRSS2 inhibitors may directly act on SARS-CoV-2 and may also have anti-inflammatory effect, reducing the transcription of proinflammatory genes.

Although the treatments described so far are based on the reduction of viral spread, other molecules exploit their immunomodulatory and anti-inflammatory effects against SARS-CoV-2 reducing cytokines. The cytokine storm contributes to the hyperactivation of $\mathrm{T}$ cells which further increases proinflammatory cytokines levels, worsening the disease related to SARS-CoV-2 infection, inducing $\mathrm{T}$ cell exhaustion, apoptosis, acute lung injury, and eventually acute respiratory distress syndrome (Channappanavar et al., 2016). In this context, IL-6 and TNF- $\alpha$ are the main proinflammatory molecules responsible for the cytokine storm that play a pivotal role in the viral infection and in associated lung damage, as demonstrated in COVID-19 patients with severe symptoms and poor prognosis (Chen X. et al., 2020; Fu et al., 2020). Also tocilizumab, a humanized monoclonal antibody, has been proposed and in some cases successfully used to halt the cytokine storm during COVID-19. Tocilizumab is used for the management of autoimmune diseases as rheumatoid arthritis that recognize a strong cytokine activation; in fact by binding the IL-6 receptor the drug is able to inhibit both classical and trans IL-6 downstream pathway (Saha et al., 2020b). In particular, a recent study showed that tocilizumab was able to reduce symptoms and improve the outcomes of patients with a severe SARS-CoV-2 infection (Dholaria et al., 2019; Xu et al., 2020). Tocilizumab has been included in the Chinese and Italian guidelines and may only be used following the acute phase of SARS-CoV-2 infection, 7 days following the beginning of symptoms, or in patients without fever and high levels of IL-6 (NHC, 2020). Among the proinflammatory cytokines, IL-1 also plays an important role in exacerbating inflammation during COVID-19 in fact, SARS$\mathrm{CoV}-2$ induces pyroptosis with the consequent release of IL- $1 \beta$ (Jiang et al., 2019). Therefore, targeting IL-1 receptor may be useful to reduce IL-1 release and cytokine storm: anakinra, for example, is a recombinant human IL-1 receptor antagonist, approved for rheumatoid arthritis, which improved the survival rate of patients with SARS-Cov-2 infection (Shakoory et al., 2016). In a recent trial on 52 COVID-19 treated with anakinra a significant reduction of mortality was observed, along with a significant decrease in the need for mechanical invasive ventilation, with no evident adverse events (Huet et al., 2020).

Besides these drugs there is a lot of literature revealing that the use of natural products was recommended for the prevention and treatment of acute respiratory syndrome (Liu et al., 2004) and four Chinese herbal medicines were suggested in the prevention program during the H1N1 influenza (National Administration of Traditional Chinese Medicine, 2009; Cragg and Newman, 2013). The application of the traditional Chinese medicine for the treatment of SARS-CoV-2 is currently based on the treatments used for SARS-CoV, thanks to their similarities (Gong et al., 2008; Nguyen et al., 2012). A retrospective observational study carried out in 293 patients affected by COVID-19 showed that the prescription of common herbs used in folk medicine may be considered as an integrative approach for COVID-19 management, since some popularly used drugs, such as moxifloxacin, oseltamivir, and Arbidol, are not always effective in reducing severe symptoms (Shu et al., 2020). However, since COVID-19 is a complex clinical disease that is often complicated by severe disorders, as clotting activation, rapid degeneration of parenchyma, and organ failure, additional studies will be requested to confirm the efficacy of traditional medicine approach alone or in combination with other drugs.

As previously described, SARS-CoV-2 infects target cells through ACE2 binding; therefore, targeting ACE2 with natural compounds might prevent SARS-CoV-2 infection. For instance, baicalin is a natural component extracted from Scutellaria 
baicalensis and its antiviral and anti-inflammatory activity have been demonstrated also during coronavirus infection. As a matter of fact, this natural molecule was proven effective against $\mathrm{H} 1 \mathrm{~N} 1$ virus and respiratory syncytial virus infection, and last but not least, a recent study demonstrated that baicalin significantly inhibited SARS-CoV S-protein binding to ACE2 (Zhang et al., 2011; Deng et al., 2012; Cheng et al., 2014). Moreover, it is worthy of interest to consider that baicalin exerts potent anti-inflammatory effects and significantly may reduce cytokines production (Li et al., 2000). Therefore, baicalin, as well as other natural products, might be considered as potential therapeutic approaches for the treatment of SARS-CoV-2 infection and the associated cytokine storm production, thus reducing the proinflammatory cytokines release and avoiding the progression of COVID-19 to a severe disease that might increase the rate of mortality. Natural products might be useful to prevent and alleviate SARS-CoV-2 infection in the near future, but in vivo and later clinical studies will be needed to investigate their possible antiviral and anti-inflammatory effects.

\section{CONCLUSION}

Although the whole world is focused on finding a cure for COVID-19 symptoms and a vaccine for SARS-CoV-2, it seems that a way out is still far; in these times collaboration and

\section{REFERENCES}

Aktas, A., Tuzun, B., Taskin, A. H., Sayin, K., and Ataseven, H. (2020). How do arbidol and its analogs inhibit the SARS-CoV-2? Bratisl. Lek. Listy 121, 705-711. doi:10.4149/BLL_2020_115

Beacon, T. H., Su, R. C., Lakowski, T. M., Delcuve, G. P., and Davie, J. R. (2020). SARS-CoV-2 multifaceted interaction with the human host. Part II: innate immunity response, immunopathology, and epigenetics. IUBMB Life 72, 2331-2354. doi:10.1002/iub.2379.

Behl, T., Kaur, I., Bungau, S., Kumar, A., Uddin, M. S., Kumar, C., et al. (2020). The dual impact of ACE2 in COVID-19 and ironical actions in geriatrics and pediatrics with possible therapeutic solutions. Life Sci. 257, 118075. doi:10.1016/ j.lfs. 2020.118075

Bordallo, B., Bellas, M., Cortez, A. F., Vieira, M., and Pinheiro, M. (2020). Severe COVID-19: what have we learned with the immunopathogenesis? $A d v$ Rheumatol 60, 50. doi:10.1186/s42358-020-00151-7

Cao, B., Wang, Y., Wen, D., Liu, W., Wang, J., Fan, G., et al. (2020). A trial of lopinavir-ritonavir in adults hospitalized with severe COVID-19. N. Engl. J. Med. 382, 1787-1799. doi:10.1056/NEJMoa2001282.

Cascella, M., Rajnik, M., Cuomo, A., Dulebohn, S. C., and Di Napoli, R. (2020). Features, evaluation and treatment coronavirus (COVID-19). Treasure Island, FL: StatPearls Publishing. StatPearls [Internet].

Chakraborty, C., Sharma, A. R., Bhattacharya, M., Sharma, G., and Lee, S. S. (2020). The 2019 novel coronavirus disease (COVID-19) pandemic: a zoonotic prospective. Asian Pac. J. Trop. Med. 13, 242-246. doi:10.4103/1995-7645. 281613

Chakraborty, C., Sharma, A. R., Bhattacharya, M., Sharma, G., Lee, S. S., and Agoramoorthy, G. (2020). Consider TLR5 for new therapeutic development against COVID-19. J. Med. Virol. 92, 1-2. doi:10.1002/jmv.25997

Chakraborty, C., Sharma, A. R., Sharma, G., Bhattacharya, M., and Lee, S. S. (2020). SARS-CoV-2 causing pneumonia-associated respiratory disorder (COVID-19): diagnostic and proposed therapeutic options. Eur. Rev. Med. Pharmacol. Sci. 24, 4016-4026. doi:10.26355/eurrev_202004_20871

Chakraborty, C., Sharma, A. R., Sharma, G., Bhattacharya, M., Saha, R. P., and Lee, S. S. (2020). Extensive partnership, collaboration, and teamwork is required to communication among governments and scientists of different countries is important and might be the only way to provide the necessary solution for this crisis (Chakraborty et al., 2020).

So far, no specific antiviral therapy or vaccine has been identified for the treatment or prevention of SARS-CoV-2 infection. Potential therapeutic approaches here discussed are focused on blocking the virus from infecting cells or on limiting the exaggerated inflammatory response related to COVID-19 to prevent severe and fatal lung injury. The numerous clinical trials that have been registered during the emergency in the various countries were not extensively reviewed by the Institutional Review Board and may suffer from important biases, as the lack of appropriate control groups, which makes it even harder to obtain a proper interpretation of the results. Nonetheless, it is important to advise that the whole scientific community has learned lots of important issues in trying to find a cure for this disease and in some cases the best clinical results have been obtained empirically using a combination of different drugs or natural products, suggesting that probably this could be the way to fight this multiorgan disease.

\section{AUTHOR CONTRIBUTIONS}

FM and NI wrote the paper; $\mathrm{AB}$ made critical revision.

stop the COVID-19 outbreak. Arch. Med. Res. 51, 728-730. doi:10.1016/j. arcmed.2020.05.021

Chan, J. F., To, K. K., Tse, H., Jin, D. Y., and Yuen, K. Y. (2013). Interspecies transmission and emergence of novel viruses: lessons from bats and birds. Trends Microbiol. 21, 544-555. doi:10.1016/j.tim.2013.05.005

Channappanavar, R., Fehr, A. R., Vijay, R., Mack, M., Zhao, J., Meyerholz, D. K., et al. (2016). Dysregulated type I interferon and inflammatory monocytemacrophage responses cause lethal pneumonia in SARS-CoV-infected mice. Version 2. Cell Host Microbe 19, 181-193. doi:10.1016/j.chom.2016.01.007

Chatterjee, S. K., Saha, S., and Munoz, M. N. M. (2020). Molecular pathogenesis, immunopathogenesis and novel therapeutic strategy against COVID-19. Front Mol Biosci 7, 196. doi:10.3389/fmolb.2020.00196

Chen, J., Lin, S., Niu, C., and Xiao, Q. (2020). Clinical evaluation of Shufeng Jiedu capsules combined with umifenovir (Arbidol) in the treatment of common-type COVID-19: a retrospective study. Expet Rev. Respir. Med. 17, 1-9. doi:10.1080/ 17476348.2020.1822741

Chen, N., Zhou, M., Dong, X., Qu, J., Gong, F., Han, Y., et al. (2020). Epidemiological and clinical characteristics of 99 cases of 2019 novel coronavirus pneumonia in Wuhan, China: a descriptive study. Lancet 395, 507-513. doi:10.1016/S0140-6736(20)30211-7

Chen, X., Zhao, B., Qu, Y., Chen, Y., Xiong, J., Feng, Y., et al. (2020). Detectable serum SARS-CoV-2 viral load (RNAaemia) is closely correlated with drastically elevated interleukin 6 (IL-6) level in critically ill COVID-19 patients. Clin. Infect. Dis. 71, 1937-1942. doi:10.1093/cid/ciaa449

Cheng, K., Wu, Z., Gao, B., and Xu, J. (2014). Analysis of influence of baicalin joint resveratrol retention enema on the TNF-alpha, SIgA, IL-2, IFN-gamma of rats with respiratory syncytial virus infection. Cell Biochem. Biophys. 70, 1305-1309. doi:10.1007/s12013-014-0055-9

Conti, P., Ronconi, G., Caraffa, A., Gallenga, C. E., Ross, R., Frydas, I., et al. (2020). Induction of pro-inflammatory cytokines (IL-1 and IL-6) and lung inflammation by coronavirus-19 (COVI-19 or SARS-CoV-2): antiinflammatory strategies. J. Biol. Regul. Homeost. Agents 14, 34. doi:10.23812/ CONTI-E

Cragg, G. M., and Newman, D. J. (2013). Natural products: a continuing source of novel drug leads. Biochim. Biophys. Acta 1830, 3670-3695. doi:10.1016/j. bbagen.2013.02.008 
Deng, Y. F., Aluko, R. E., Jin, Q., Zhang, Y., and Yuan, L. J. (2012). Inhibitory activities of baicalin against renin and angiotensin-converting enzyme. Pharm. Biol. 50, 401-406. doi:10.3109/13880209.2011.608076

Dholaria, B. R., Bachmeier, C. A., and Locke, F. (2019). Mechanisms and management of chimeric antigen receptor T-cell therapy-related toxicities. BioDrugs 33, 45-60. doi:10.1007/s40259-018-0324-z

Du Toit, A. (2020). Outbreak of a novel coronavirus. Nat. Rev. Microbiol. 18, 123. doi:10.1038/s41579-020-0332-0

Fu, Y., Cheng, Y., and Wu, Y. (2020). Understanding SARS-CoV-2-mediated inflammatory responses: from mechanisms to potential therapeutic tools. Virol. Sin. 3, 1-6. doi:10.1007/s12250-020-00207-4

Fung, T. S., and Liu, D. X. (2019). Human coronavirus: host-pathogen interaction. Annu. Rev. Microbiol. 73, 529-557. doi:10.1146/annurev-micro-020518-115759

Glowacka, I., Bertram, S., Herzog, P., Pfefferle, S., Steffen, I., Muench, M. O., et al. (2010). Differential downregulation of ACE2 by the spike proteins of severe acute respiratory syndrome coronavirus and human coronavirus NL63. J. Virol. 84, 1198-1205. doi:10.1128/JVI.01248-09

Gong, S. J., Su, X. J., Yu, H. P., Li, J., Qin, Y. J., Xu, Q., et al. (2008). A study on antiSARS-CoV 3CL protein of flavonoids from litchi chinensis sonn core. Chin. Pharmacol. Bull. 24, 699-700.

Hoffmann, M., Kleine-Weber, H., Schroeder, S., Krüger, N., Herrler, T., Erichsen, S., et al. (2020). SARS-CoV-2 cell entry depends on ACE2 and TMPRSS2 and is blocked by a clinically proven protease inhibitor. Cell 181, 271-280. doi:10. 1016/j.cell.2020.02.052

Holshue, M. L., DeBolt, C., Lindquist, S., Lofy, K. H., Wiesman, J., and Bruce, H. (2020). First case of 2019 novel coronavirus in the United States. N. Engl. J. Med. 382, 929-936. doi:10.1056/NEJMoa2001191

Huang, C., Wang, Y., Li, X., Ren, L., Zhao, J., Hu, Y., et al. (2020). Clinical features of patients infected with 2019 novel coronavirus in Wuhan, China. Lancet 395, 497-506. doi:10.1016/S0140-6736(20)30183-5

Huet, T., Beaussier, H., Voisin, O., Jouveshomme, S., Dauriat, G., Lazareth, I., et al. (2020). Anakinra for severe forms of COVID-19: a cohort study. Lancet 2, E393-E400. doi:10.1016/S2665-9913(20)30164-8

Hung, I. F., Lung, K. C., Tso, E. Y., Liu, R., Chung, T. W., Chu, M. Y., et al. (2020). Triple combination of interferon beta- $1 \mathrm{~b}$, lopinavir-ritonavir, and ribavirin in the treatment of patients admitted to hospital with COVID-19: an open-label, randomised, phase 2 trial. Lancet 395, 1695-1704. doi:10.1016/S0140-6736(20)31042-4

Imai, Y., Kuba, K., Rao, S., Huan, Y., Guo, F., Guan, B., et al. (2005). Angiotensinconverting enzyme 2 protects from severe acute lung failure. Nature 436, 112-116. doi:10.1038/nature03712

Iwasaki, A., and Medzhitov, R. (2015). Control of adaptive immunity by the innate immune system. Nat. Immunol. 16, 343-353. doi:10.1038/ni.3123

Jiang, Y., Li, J., Teng, Y., Sun, H., Tian, G., He, L., et al. (2019). Complement receptor C5aR1 inhibition reduces pyroptosis in hDPP4-transgenic mice infected with MERS-CoV. Viruses 11, 39. doi:10.3390/v11010039

Jordan, P. C., Stevens, S. K., and Deval, J. (2018). Nucleosides for the treatment of respiratory RNA virus infections. Antivir. Chem. Chemother. 26, 2040206618764483. doi:10.1177/2040206618764483

Kagan, J. C., Magupalli, V. G., and Wu, H. (2014). SMOCs: supramolecular organizing centres that control innate immunity. Nat. Rev. Immunol. 14, 821-826. doi:10.1038/nri3757

Kawai, T., and Akira, S. (2010). The role of pattern-recognition receptors in innate immunity: update on Toll-like receptors. Nat. Immunol. 11, 373-384. doi:10. 1038/ni.1863

Kumar, H., Kawai, T., and Akira, S. (2009). Toll-like receptors and innate immunity. Biochem. Biophys. Res. Commun. 388, 621-625. doi:10.1016/j. bbrc.2009.08.062

Lei, J., Kusov, Y., and Hilgenfeld, R. (2018). Nsp3 of coronaviruses: structures and functions of a large multi-domain protein. Antivir. Res. 149, 58-74. doi:10.1016/ j.antiviral.2017.11.001

Li, G., and De Clercq, E. (2020). Therapeutic options for the 2019 novel coronavirus (2019-nCoV). Nat. Rev. Drug Discov. 19, 149-150. doi:10.1038/ d41573-020-00016-0

Li, B. Q., Fu, T., Gong, W. H., Dunlop, N., Kung, H., Yan, Y., et al. (2000). The flavonoid baicalin exhibits anti-inflammatory activity by binding to chemokines. Immunopharmacology 49, 295-306. doi:10.1016/s0162-3109(00) 00244-7
Li, F., Li, W. H., Farzan, M., and Harrison, S. C. (2005). Structure of SARS coronavirus spike receptor- binding domain complexed with receptor. Science 309, 1864-1868. doi:10.1126/science.1116480

Li, S. W., Wang, C. Y., Jou, Y. J., Huang, S. H., Hsiao, L. H., Wan, L., et al. (2016). SARS coronavirus papain-like protease inhibits the TLR7 signaling pathway through removing lys63-linked polyubiquitination of TRAF3 and TRAF6. Int. J. Mol. Sci. 5, 17. doi:10.3390/ijms17050678

Li, Y., Chen, M., Cao, H., Zhu, Y., Zheng, J., and Zhou, H. (2013). Extraordinary GU-rich single-strand RNA identified from SARS coronavirus contributes an excessive innate immune response. Microb. Infect. 15, 88-95. doi:10.1016/j. micinf.2012.10.008

Liu, J., Manheimer, E., Shi, Y., and Gluud, C. (2004). Chinese herbal medicine for severe acute respiratory syndrome: a systematic review and meta-analysis. J. Alternat. Compl. Med. 10, 1041-1051. doi:10.1089/acm.2004.10.1041

Liu, L., Wei, Q., Lin, Q., Fang, J., Wang, H., Kwok, H., et al. (2019). Anti-spike IgG causes severe acute lung injury by skewing macrophage responses during acute SARS-CoV infection. JCI Insight 21, 4. doi:10.1172/jci.insight.123158

Martinon, F. (2010). Signaling by ROS drives inflammasome activation. Eur. J. Immunol. 40, 616-619. doi:10.1002/eji.200940168

Martinon, F., Burns, K., and Tschopp, J. (2002). The inflammasome: a molecular platform triggering activation of inflammatory caspases and processing of proIL-beta. Mol. Cell 10, 417-426. doi:10.1016/s1097-2765(02)00599-3

Mehra, M. R., Desai, S. S., Ruschitzka, F., and Patel, A. N. (2020). Hydroxychloroquine or chloroquine with or without a macrolide for treatment of COVID-19: a multinational registry analysis. Lancet 22, S0140-S6736. doi:10.1016/S0140-6736(20)31180-6

Moreno-Eutimio, M. A., López-Macías, C., and Pastelin-Palacios, R. (2020). Bioinformatic analysis and identification of single-stranded RNA sequences recognized by TLR7/8 in the SARS-CoV-2, SARS-CoV, and MERS-CoV genomes. Microb. Infect. 22, 226-229. doi:10.1016/j.micinf.2020.04.009

National Administration of Traditional Chinese Medicine (2009). Prevention program of traditional Chinese medicine for $2009 \mathrm{H} 1 \mathrm{~N} 1$ influenza. Chin Comm Doct. (Chin) 25, 13.

Nguyen, T. T. H., Woo, H. J., Kang, H. K., Nguyen, V. D., Kim, Y. M., Kim, D. W., et al. (2012). Flavonoid-mediated inhibition of SARS coronavirus 3C-like protease expressed in Pichia pastoris. Biotechnol. Lett. 34, 831-838. doi:10. 1007/s10529-011-0845-8

NHC (2020). Interpretation of "new coronavirus pneumonia diagnosis and treatment scheme (trial version 7)” 2020; SIMIT: vademecum per la cura delle persone con malattia da COVI-19, Sezione Regione Lombardia: Societ grave a Italiana di Malattie Infettive e Tropicali.

Richardson, P., Griffin, I., Tucker, C., Smith, D., Oechsle, O., Phelan, A., et al. (2020). Baricitinib as potential treatment for $2019-\mathrm{nCoV}$ acute respiratory disease. Lancet 395, 30-31. doi:10.1016/S0140-6736(20)30304-4

Saha, A., Sharma, A. R., Bhattacharya, M., Sharma, G., Lee, S. S., and Chakraborty, C. (2020a). Probable molecular mechanism of remdesivir for the treatment of COVID-19: need to know more. Arch. Med. Res. 51, 585-586. doi:10.1016/j. arcmed.2020.05.001

Saha, A., Sharma, A. R., Bhattacharya, M., Sharma, G., Lee, S. S., and Chakraborty, C. (2020b). Tocilizumab: a therapeutic option for the treatment of cytokine storm syndrome in COVID-19. Arch. Med. Res. 51, 595-597. doi:10.1016/j. arcmed.2020.05.009

Savarino, A., Di Trani, L., Donatelli, I., Cauda, R., and Cassone, A. (2006). New insights into the antiviral effects of chloroquine. Lancet Infect. Dis. 6, 67-69. doi:10.1016/S1473-3099(06)70361-9

Shakoory, B., Carcillo, J. A., Chatham, W. W., Amdur, R. L., Zhao, H., Dinarello, C. A., et al. (2016). Interleukin-1 receptor blockade is associated with reduced mortality in sepsis patients with features of macrophage activation syndrome: reanalysis of a prior phase III trial. Crit. Care Med. 44, 275-281. doi:10.1097/ CCM.0000000000001402

Shang, J., Ye, G., Shi, K., Wan, Y., Luo, C., Aihara, H., et al. (2020). Structural basis of receptor recognition by SARS-CoV-2. Nature 581, 221-224. doi:10.1038/ s41586-020-2179-y.

Shu, Z., Zhou, Y., Chang, K., Liu, J., Min, X., Zhang, Q., et al. (2020). Clinical features and the traditional Chinese medicine therapeutic characteristics of 293 COVID-19 inpatient cases. Front. Med. 1-16. doi:10.1007/s11684-0200803-8 
Song, W., Gui, M., Wang, X., and Xiang, Y. (2018). Cryo-EM structure of the SARS coronavirus spike glycoprotein in complex with its host cell receptor ACE2. PLoS Pathog. 14, e1007236. doi:10.1371/journal.ppat.1007236

Song, Y., Zhang, M., Yin, L., Wang, K., Zhou, Y., Zhou, M., et al. (2020). COVID-19 treatment: close to a cure? A rapid review of pharmacotherapies for the novel coronavirus (SARS-CoV-2). Int. J. Antimicrob. Agents 56, 106080. doi:10.1016/ j.ijantimicag. 2020.106080

Stebbing, J., Phelan, A., Griffin, I., Tucker, C., Oechsle, O., Smith, D., et al. (2020). COVID-19: combining antiviral and anti-inflammatory treatments. Lancet Infect. Dis. 20, 400-402. doi:10.1016/S1473-3099(20)30132-8

Takeuchi, O., and Akira, S. (2010). Pattern recognition receptors and inflammation. Cell 140, 805-820. doi:10.1016/j.cell.2010.01.022

Verdecchia, P., Cavallini, C., Spanevello, A., and Angeli, F. (2020). The pivotal link between ACE2 deficiency and SARS-CoV-2 infection. Eur. J. Intern. Med. 76, 14-20. doi:10.1016/j.ejim.2020.04.037

Vincent, M. J., Bergeron, E., Benjannet, S., Erickson, B. R., Rollin, P. E., Ksiazek, T. G., et al. (2005). Chloroquine is a potent inhibitor of SARS coronavirus infection and spread. Virol. J. 2, 69. doi:10.1186/1743-422X-2-69

Wang, S., Guo, F., Liu, K., Wang, H., Rao, S., Yang, P., et al. (2008). Endocytosis of the receptor-binding domain of SARS-CoV spike protein together with virus receptor ACE2. Virus Res. 136, 8-15. doi:10.1016/j. virusres.2008.03.004

Wang, Y., Zhang, D., Du, G., Du, R., Zhao, J., Jin, Y., et al. (2020). Remdesivir in adults with severe COVID-19: a randomised, double-blind, placebo-controlled, multicentre trial. Lancet 395, 1569-1578. doi:10.1016/S0140-6736(20)31022-9

Wong, C. K., Lam, C. W., Wu, A. K., Ip, W. K., Lee, N. L., Chan, I. H., et al. (2004). Plasma inflammatory cytokines and chemokines in severe acute respiratory syndrome. Clin. Exp. Immunol. 136, 95-103. doi:10.1111/j.1365-2249.2004.02415.x

Xu, X., Han, M., Li, T., Sun, W., Wang, D., Fu, B., et al. (2020). Effective treatment of severe COVID-19 patients with tocilizumab. Proc. Natl. Acad. Sci. U.S.A. 117, 10970-10975. doi:10.1073/pnas.2005615117
Yan, Y., Zou, Z., Sun, Y., Li, X., Xu, K. F., Wei, Y., et al. (2013). Anti-malaria drug chloroquine is highly effective in treating avian influenza A H5N1 virus infection in an animal model. Cell Res. 23, 300-302. doi:10.1038/cr. 2012.165

Yang, M. (2020). Cell pyroptosis, a potential pathogenic mechanism of 2019-nCoV infection. SSRN 7. doi:10.2139/ssrn. 3527420

Yu, J. S., Tseng, C. K., Lin, C. K., Hsu, Y. C., Wu, Y. H., Hsieh, C. L., et al. (2017). Celastrol inhibits dengue virus replication via up-regulating type I interferon and downstream interferon-stimulated responses. Antivir. Res. 137, 49-57. doi:10.1016/j.antiviral.2016.11.010

Zhang, C. J., Gu, L. G., and Yu, H. T. (2011). Antagonism of baicalin on cell cyclical distribution and cell apoptosis in A549 cells infected with influenza A (H1N1) virus. Bing Du Xue Bao 27, 108-116.

Zhang, C. J., Gu, L. G., and Yu, H. T. (2011). Antagonism of baicalin on cell cyclical distribution and cell apoptosis in A549 cells infected with influenza A (H1N1) virus. Bing Du Xue Bao 27, 108-116.

Zhang, L., Zhang, F., Yu, W., He, T., Yu, J., Yi, C. E., et al. (2006). Antibody responses against SARS coronavirus are correlated with disease outcome of infected individuals. J. Med. Virol. 78, 1-8. doi:10.1002/jmv.20499

Conflict of Interest: The authors declare that the research was conducted in the absence of any commercial or financial relationships that could be construed as a potential conflict of interest.

Copyright (c) 2020 Mannino, Bitto and Irrera. This is an open-access article distributed under the terms of the Creative Commons Attribution License (CC $B Y)$. The use, distribution or reproduction in other forums is permitted, provided the original author(s) and the copyright owner(s) are credited and that the original publication in this journal is cited, in accordance with accepted academic practice. No use, distribution or reproduction is permitted which does not comply with these terms. 